\title{
17th International Chromosome Conference
}

(C) Springer Science + Business Media B.V. 2009

Organizing Committee:

Ken Shull - Appalachian State University, Local Coordinator

Lorinda Anderson - Colorado State University, Program Chair

Terry Ashley - Yale University School of Medicine (retired)

Stephen Stack - Colorado State University

Dwayne Wise - Mississippi State University

The 17th meeting of the International Chromosome Conference (ICC) will take place June 23-June 26, 2009 in Boone, North Carolina. Boone is the home of Appalachian State University and was the setting for four chromosome conferences that were held in the 1980's and early 1990's. The International Chromosome Conference series began in 1964 under the title Oxford Chromosome Conference. From 1964 to 1974, the meetings were held every two years. The meetings were then changed to a three year schedule. Recently, the conference was changed back to the historical two-year schedule. This ICC will be the first to be held in the United States.

Chromosome biology is a dynamic field that has experienced significant growth over the last decade with technological advances in fluorescent in situ hybridization, light and electron microscopic techniques, and faster and cheaper DNA sequencing. Indeed, one of the main challenges for the future will be putting this large mass of DNA sequence data into its proper biological context as chromatin within chromosomes. This type of integration will have a major impact on many fields related to chromosome biology such as gene expression, recombination, chromatin packaging, chromosome structure and the evolution of genomes and chromosomes. The ICC has long considered chromosome biology to be broadly inclusive and to include both structural and functional aspects of chromatin and chromosomes. Topics at the $17^{\text {th }}$ ICC include advances in imaging and molecular technology, centromeres and neocentromeres, telomeres, heterochromatin and repeated sequences, epigenetics and gene expression, nuclear architecture, chromatin and chromosome structure, polyploidy and aneuploidy, specialized (e.g., sex, B, and artificial) chromosomes, chromosomes in genome sequencing and analysis, chromosome and genome evolution, and meiosis and recombination. The International Chromosome Conferences are unusual in that researchers using both plant and animal models are included, thus presenting a valuable occasion for new and fruitful interactions between researchers pursuing similar questions using different model systems.

The organizers thank the plenary speakers, Scott Hawley (Stowers Institute for Medical Research, Kansas City) and Jennifer Graves (Austrialian National University, Canberra), who will speak on the molecular biology of meiosis and the evolution of sex chromosomes, respectively. We especially thank the session leaders for contributing their time and energy to arrange an outstanding slate of speakers for the conference. We also thank the National Science Foundation for providing support for students. 